\title{
ON STABLE COMPLETE HYPERSURFACES WITH VANISHING $r$-MEAN CURVATURE
}

\author{
Manfredo do CARmo And Maria F. Elbert
}

(Received July 10, 2002, revised August 22, 2003)

\begin{abstract}
A form of Bernstein theorem states that a complete stable minimal surface in euclidean space is a plane. A generalization of this statement is that there exists no complete stable hypersurface of an $n$-euclidean space with vanishing $(n-1)$-mean curvature and nowhere zero Gauss-Kronecker curvature. We show that this is the case, provided the immersion is proper and the total curvature is finite.
\end{abstract}

1. Introduction. Let $x: M^{n} \rightarrow \boldsymbol{R}^{n+1}$ be a hypersurface of the $(n+1)$-euclidean space $\boldsymbol{R}^{n+1}$. We assume that $M=M^{n}$ is orientable and fix an orientation for $M$. Let $g: M \rightarrow$ $S_{1}^{n} \subset \boldsymbol{R}^{n+1}$ be the Gauss map in the given orientation, where $S_{1}^{n}$ is the unit $n$-sphere in $\boldsymbol{R}^{n+1}$. Recall that the linear operator $A: T_{p} M \rightarrow T_{p} M, p \in M$, associated to the second fundamental form of $x$ is given by

$$
\langle A(X), Y\rangle=-\left\langle\bar{\nabla}_{X} N, Y\right\rangle, \quad X, Y \in T_{p} M,
$$

where $\bar{\nabla}$ is the covariant derivative of the ambient space and $N$ is the unit normal vector of $x$ in the given orientation. The map $A=-d g$ is self-adjoint and its eigenvalues are the principal curvatures $k_{1}, k_{2}, \ldots, k_{n}$ of $x$.

Assume now that the immersion is complete. We will say that the total curvature of the immersion is finite if $\int_{M}|A|^{n} d M<\infty$, where $|A|=\left(\sum_{i} k_{i}^{2}\right)^{1 / 2}$.

Consider now the elementary symmetric functions $S_{r}, r=0,1, \ldots, n$, of the principal curvatures $k_{1}, \ldots, k_{n}$ of $x$ :

$$
S_{0}=1, \quad S_{r}=\sum_{i_{1}<\cdots<i_{r}} k_{i_{1}} \ldots k_{i_{r}}, \quad i_{1}, \ldots, i_{r}=1, \ldots, n,
$$

and their associated $r$-mean curvatures $H_{r}$ given by

$$
H_{r}=\left(\begin{array}{l}
n \\
r
\end{array}\right)^{-1} S_{r} .
$$

Hypersurfaces in euclidean spaces with $H_{r}=0$ generalize minimal hypersurfaces $\left(H_{1}=\right.$ $0)$. The relation is even deeper, since minimal hypersurfaces are critical points of the functional $A_{0}=\int_{M} H_{0} d M$ for compactly supported variations of $M$, whereas hypersurfaces with $H_{r+1}=0$ are critical points of the functional $A_{r}=\int_{M} H_{r} d M$ also for compactly supported variations [11]. A breakthrough in the study of such hypersurfaces was made when Hounie

2000 Mathematics Subject Classification. Primary 53C42.

Key words and phrases. Stability, $r$-mean curvature, complete, finite total curvature. 
and Leite [8] proved that the equation $H_{r+1}=0, r \neq 0, n-1$, is elliptic provided that rank $A>r$. In the case $r=0$, no such condition is necessary, since the equation of a minimal hypersurface is automatically elliptic.

In [1], a definition of stability was given for hypersurfaces of the euclidean space with $H_{r+1}=0$ (see Section 2 for details) and the following theorems were proved for the special case where $r+1=n-1$ (in this case, it is not difficult to see that the condition rank $A>r$ is equivalent to $H_{n} \neq 0$ everywhere).

THEOREM A (Theorem 1.2 of [1]). Let $x: M^{n} \rightarrow \boldsymbol{R}^{n+1}$ be an orientable hypersurface with $H_{n-1}=0$ and $H_{n} \neq 0$ everywhere. Let $D \subset M$ be a bounded domain with piecewise smooth boundary. Assume that

$$
\text { Area of } g(D)<\text { Area of a hemisphere of } S_{1}^{n} \text {. }
$$

Then $D$ is stable and the estimate is sharp.

THEOREM B (Corollary 1.7 of [1]). Let $x: M^{n} \rightarrow \boldsymbol{R}^{n+1}$ and $D \subset M$ be as in Theorem A. Assume that the Gauss map g restricted to $\bar{D}$ is a covering map onto $g(\bar{D})$, and that the first eigenvalue $\lambda_{1}(g(D))$ of $g(D)$ for the spherical Laplacian satisfies $\lambda_{1}(g(D))<n$. Then $D$ is unstable.

Theorem A generalizes a theorem of Barbosa and do Carmo (Theorem 1.3 of [2]), which gives a condition for stability of bounded domains of orientable minimal surfaces in $\boldsymbol{R}^{3}$, and Theorem B generalizes a theorem of A. Schwarz (see [2], Theorem 2.7) for instability of similar domains.

The question naturally arises of what can be said about hypersurfaces $x: M^{n} \rightarrow \boldsymbol{R}^{n+1}$ with $H_{n-1}=0$ and $H_{n} \neq 0$ everywhere that are orientable, complete and stable in the sense that every bounded domain in $M$ is stable. This is a very strong condition, and, in the minimal case, it has been proved that the only orientable, complete stable minimal surface in $\boldsymbol{R}^{3}$ is the plane (see [5] and [7]).

Based on the above considerations, in [1] the following conjecture was proposed. There exists no complete, orientable, stable hypersurface $x: M^{n} \rightarrow \boldsymbol{R}^{n+1}$ with $H_{n-1}=0$ and $H_{n} \neq 0$ everywhere. Here we show that with some additional conditions the conjecture is true. Namely, we prove

THEOREM 1.1. There exists no complete orientable, proper, stable hypersurface $x: M^{n} \rightarrow \boldsymbol{R}^{n+1}, n \geq 3$, with $H_{n-1}=0$ and $H_{n} \neq 0$ everywhere and of finite total curvature.

2. Proof of Theorem 1.1. Before going into the proof of Theorem 1.1, we need to fix some notation and to recall relevant facts on stability. Further details can be found in [11], [12], [3] and [1].

Let $x: M^{n} \rightarrow \boldsymbol{R}^{n+1}$ be an orientable hypersurface with $H_{r+1}=0$. A regular domain $D \subset M$ is a domain with compact closure and piecewise smooth boundary. We say that $D$ is stable if either $A_{r}^{\prime \prime}(0)>0$ for all variations with compact support in $D$ or $A_{r}^{\prime \prime}(0)<0$ for all 
such variations. A justification for this definition can be found in the Introduction of [1]. If for some variation with compact support in $D$ we have $A_{r}^{\prime \prime}(0)>0$, while for some other such variation, we have $A_{r}^{\prime \prime}(0)<0$, we say that $D$ is unstable.

Following [11], we define a linear map $P_{r}$ of $T_{p} M$ by

$$
P_{0}=I, \quad P_{r}=S_{r} I-A P_{r-1},
$$

where $I$ is the identity matrix and $A$ is the linear map defined in the Introduction. Next, we define a second order linear operator $L_{r}$ by

$$
L_{r} f=\operatorname{div}\left(P_{r} \nabla f\right),
$$

where $\nabla f$ is the gradient of $f$. We then write the Jacobi equation of the variational problem that defines the hypersurfaces with $H_{r+1}=0$ :

$$
T_{r} f \stackrel{\text { def }}{=} L_{r} f-(r+2) S_{r+2} f=0 .
$$

The Jacobi equation (2) is the linearization of the equation $H_{r+1}=0$. As we mentioned in the Introduction, $H_{n} \neq 0$ everywhere is a sufficient condition for (2) to be elliptic. By (1), this is equivalent to the fact that $P_{r}$ has all its eigenvalues of the same sign. We denote by $\theta_{i}(r)$ the eigenvalues of $\sqrt{P_{r}} A$ when $P_{r}$ is positive definite, and the eigenvalues of $\sqrt{-P_{r}} A$ when $P_{r}$ is negative definite. We will assume for convenience that $P_{r}$ is positive definite, leaving the details of the other case to the reader.

With this notation, we can rewrite the Jacobi operator $T_{r}$ as ([1], Section 2)

$$
T_{r}=L_{r}+\left\|\sqrt{P_{r}} A\right\|^{2},
$$

where $\left\|\sqrt{P_{r}} A\right\|^{2}=\sum_{i} \theta_{i}^{2}(r)$. Finally, we define the Morse index form $I_{r}$ of our variational problem as

$$
I_{r}(f, g)=-\int_{M} f T_{r}(g) d M .
$$

In the case $r+1=n-1$, i.e., $H_{n-1}=0$, it can be shown that ([1], Lemma 2.4)

$$
\left(\theta_{i}(n-2)\right)^{2}=-S_{n} .
$$

In the proof of our theorem, we are going to use Theorems A and B of the Introduction. Concerning Theorem A, it should be noticed that the fact that the area of $g(D)$ is smaller than the area of a hemisphere of $S_{1}^{n}$ implies, by symmetrization, that $\lambda_{1}(g(D))>n$, and the latter is what is used in the proof of Theorem 1.2 of [1]. Thus, Theorem $\mathrm{A}^{\prime}$ below holds:

THEOREM $\mathrm{A}^{\prime}$. Let $x: M^{n} \rightarrow \boldsymbol{R}^{n+1}$ and $D \subset M$ be as in Theorem $\mathrm{A}$ of the Introduction. Assume that $\lambda_{1}(g(D))>n$. Then $D$ is stable.

We also need a lemma which is proved in [1] (Lemma 2.7) and that will be quoted here as Lemma A. We use $C_{0}^{\infty}(D)$ to denote the space of differentiable functions that vanish on the boundary $\partial D$ of a regular domain $D$, and $C_{c}^{\infty}(D)$ to denote those differentiable functions that have a (compact) support in $D$.

LEMMA A ([1], see also [14]). The following statements are equivalent: 
(i) There exists $f \in C_{c}^{\infty}(D)$ such that $I_{r}(f, f) \leq 0$.

(ii) There exists $f \in C_{c}^{\infty}(D)$ such that $I_{r}(f, f)<0$.

(iii) There exists $f \in C_{0}^{\infty}(D)$ such that $I_{r}(f, f)<0$.

We still need a definition. We say that the boundary $\partial D$ of a regular domain $D$ is $a$ first conjugate boundary if there exists a Jacobi field that vanishes on $\partial D$ and there exists no Jabobi field that vanishes in (the open set) D. A Jacobi field $f N$ is a normal vector field such that $f$ satisfies the Jacobi equation (2).

Let $D$ be a domain such that $\partial D$ is a first conjugate boundary. We observe that every domain properly contained in $D$ is stable and every domain that contains $D$ properly is unstable. In fact, if $D^{\prime} \varsubsetneqq D$ is not stable, there exists $f \in C_{c}^{\infty}\left(D^{\prime}\right)$ such that $I_{r}(f, f) \leq 0$. By Lemma A, there exists $f \in C_{0}^{\infty}\left(D^{\prime}\right)$ such that $I_{r}(f, f)<0$. By the Morse Index Theorem, there exists $D^{\prime \prime} \subset D^{\prime}$ and a Jacobi field vanishing on $\partial D^{\prime \prime}$. This is a contradiction and proves the first part of the statement.

To prove the second part of the statement, let $D^{\prime \prime} \supseteq D$. Since $\partial D$ is a conjugate boundary, by the Morse Index Theorem there exists $f \in C_{0}^{\infty}\left(D^{\prime \prime}\right)$ with $I_{r}(f, f)<0$. By Lemma A, there exists $f \in C_{c}^{\infty}\left(D^{\prime \prime}\right)$ with $I_{r}(f, f)<0$, hence $D^{\prime \prime}$ is unstable.

REMARK 2.1. Although we have no need of it, it is not hard to show that the two-part statement that we just proved is an equivalent definition of a first conjugate boundary.

The proof of Theorem 1.1 will depend on Lemmas 2.2, 2.3 and 2.5 below.

LEMMA 2.2. Let $x: M^{n} \rightarrow \boldsymbol{R}^{n+1}$ be an orientable hypersurface with $H_{n-1}=0$ and $H_{n} \neq 0$ everywhere. Assume that its Gauss map $g: M^{n} \rightarrow S^{n}$ is injective. Let $D \subset M$ be a regular domain such that $\partial D$ is a first conjugate boundary. Then the following hold.

(a) The first eigenvalue $\lambda_{1}(g(D))$ for the spherical Laplacian satisfies $\lambda_{1}(g(D))=n$.

(b) Let $f: g(D) \rightarrow \boldsymbol{R}$ be the first eigenfunction of $g(D)$. Then $u=f \circ g$ satisfies the Jacobi equation, $u>0$ in $D$ and $u=0$ on $\partial D$.

Proof. We will prove (a). Indeed, $\lambda_{1}(g(D))$ is not smaller than $n$. Otherwise, we could find a domain $D^{\prime} \subset D$ such that $\lambda_{1}\left(g\left(D^{\prime}\right)\right)<n$. Thus $D^{\prime}$ is unstable by Theorem B and this contradicts the fact that every domain contained in $D$ is stable (since $\partial D$ is a first conjugate boundary). Also, it cannot occur that $\lambda_{1}(g(D))>n$. Otherwise, we could find a domain $D^{\prime \prime} \supset D$ such that $\lambda_{1}\left(g\left(D^{\prime \prime}\right)\right)>n$. By Theorem $\mathrm{A}^{\prime}, D^{\prime \prime}$ is stable, and this is a contradiction. Thus $\lambda_{1}(g(D))=n$ and this proves (a).

We now prove (b). Since the Gauss map is injective, $\partial(g(D))=g(\partial D)$, and then

$$
\tilde{\Delta} u+n u=0, \quad u>0 \text { in } D, \quad u=0 \text { on } \partial D,
$$

where $\tilde{\Delta}$ is the Laplacian of the pullback metric $\langle\langle\rangle$,$\rangle on M$ by $g$ (we recall that $H_{n} \neq 0$ ). By Stokes theorem, 


$$
0=\int_{D}\left(\|\tilde{\nabla} u\|^{2}-n u^{2}\right) d S=\int_{D}\left(\|\tilde{\nabla} u\|^{2}-n u^{2}\right)\left|S_{n}\right| d M,
$$

where $d M$ and $d S=\left|S_{n}\right| d M$ are the volume elements of the induced metric and the pullback metric, respectively.

For notational simplicity, we write $\left(\theta_{i}(n-2)\right)^{2}=\theta_{i}^{2}$. Since, by (3), $\theta_{i}^{2}=-S_{n}$, we have, assuming that $P_{r}$ is positive definite,

$$
\frac{1}{n}\left\|\sqrt{P_{r}} A\right\|^{2}=\frac{1}{n} \sum_{j} \theta_{j}^{2}=\left|S_{n}\right| .
$$

Also, denoting by $\lambda_{i}$ the eigenvalues of

$$
\frac{\sqrt{P_{r}} A}{\left\|\sqrt{P_{r}} A\right\|}
$$

we obtain that

$$
\lambda_{i}^{2}=\frac{\theta_{i}^{2}}{\sum_{j} \theta_{j}^{2}}=\frac{1}{n} .
$$

Since, for any $X \in T_{p} M$,

$$
\|X\|^{2}=\left\|\left(\frac{\sqrt{P_{r}} A}{\left\|\sqrt{P_{r}} A\right\|}\right)^{-1}\right\|^{2}\left\|\frac{\sqrt{P_{r}} A}{\left\|\sqrt{P_{r}} A\right\|} X\right\|^{2},
$$

we can write

Therefore, we have from (4),

$$
\|\tilde{\nabla} u\|^{2}=n \frac{\left\|\sqrt{P_{r}} A \tilde{\nabla} u\right\|^{2}}{\left\|\sqrt{P_{r}} A\right\|^{2}} .
$$

$$
\begin{aligned}
0 & =\int_{D}\left(\frac{n\left\|\sqrt{P_{r}} A \tilde{\nabla} u\right\|^{2}}{\left\|\sqrt{P_{r}} A\right\|^{2}}-n u^{2}\right) \frac{1}{n}\left\|\sqrt{P_{r}} A\right\|^{2} d M \\
& =\int_{D}\left(\left\|\sqrt{P_{r}} A \tilde{\nabla} u\right\|^{2}-\left\|\sqrt{P_{r}} A\right\|^{2} u^{2}\right) d M .
\end{aligned}
$$

By using that $\tilde{\nabla}=A^{-2} \nabla$ ([1], Lemma 2.9), that $P_{r}$ commutes with $A$, and that $\left\langle\left\langle A^{-1} X, A^{-1} X\right\rangle\right\rangle=\langle X, X\rangle$, we have, by Stokes Theorem,

$$
0=\int_{D}\left(\left|\sqrt{P_{r}} \nabla u\right|^{2}-\left\|\sqrt{P_{r}} A\right\|^{2} u^{2}\right) d M=I_{r}(u, u) .
$$

Now we use that $\partial D$ is a first conjugate boundary. Thus for every $\varphi \in C_{0}^{\infty}(D)$, we have that $I_{r}(\varphi, \varphi) \geq 0$. Otherwise, there exists $g \in C_{0}^{\infty}(D)$ with $I_{r}(g, g)<0$; by the Morse Index Theorem, there exists a Jacobi field in $D^{\prime} \mp D$ vanishing in $\partial D^{\prime}$, and this is a contradiction. Then, for any $v \in C_{0}^{\infty}(D)$, we obtain for all $t \in \boldsymbol{R}$,

$$
0 \leq I_{r}(u+t v, u+t v)=2 t I_{r}(u, v)+t^{2} I_{r}(v, v) .
$$

Hence $I_{r}(u, v)=0$, and thus $u$ satisfies the Jacobi equation. This proves (b) and completes the proof of Lemma 2.2. 
LEMMA 2.3. Let $S_{1}^{n} \subset \boldsymbol{R}^{n+1}$ be the unit sphere of $\boldsymbol{R}^{n+1}$ and $p=(0, \ldots, 0,1) \in$ $S_{1}^{n}$. Then there exist a domain $D$, symmetric relative to the equator of $S_{1}^{n}$, and a function $f: S_{1}^{n} \rightarrow \boldsymbol{R}$ such that $\lambda_{1}(D)=n$ and that $f$ is the first eigenfunction of $D$. Furthermore, $\lim _{q \rightarrow \pm p} f(q)=-\infty, q \in S_{1}^{n}$, where $-p$ is the antipodal point to $p$.

PROOF. This is an application of Lemma 2.2 to rotation hypersurfaces. Let $\boldsymbol{R}^{n+1}$ have coordinates $x_{1}, \ldots, x_{n+1}=y$. Following [10], we let $O x_{1}$ be the axis of rotation and let $y=$ $h\left(x_{1}\right)$ be the equation of the generating curve $C$ of the rotation hypersurface $x: M^{n} \rightarrow \boldsymbol{R}^{n+1}$ with $H_{n-1}=0$. It is easily checked that $H_{n} \neq 0$ everywhere for such hypersurfaces and that the curve $C$ is symmetric.

Now consider the domain $W \subset M$ bounded by the rotation of the points of contact of the tangent lines to $C$ issued from the origin 0 of $\boldsymbol{R}^{n+1}$. It is known ([1], §3.7) that the support function $\langle x, N\rangle$ satisfies the Jacobi equation, is positive in $W$ and vanishes in $\partial W$. Thus $\partial W$ is a first conjugate boundary and, since the Gauss map of such rotation hypersurfaces is injective ([10], §2), Lemma 2.2 implies that the symmetric domain $D=g(W) \subset S_{1}^{n}$ satisfies $\lambda_{1}(D)=n$. Furthermore, if $f$ is the first eigenfunction of $D$, then, again by Lemma 2.2, $u=f \circ g$ satisfies the Jacobi equation, $u>0$ in $W$ and $u=0$ in $\partial W$. It follows that $u=\langle x, N\rangle$.

Since $M$ behaves asymptotically like a parabola ([10], §2), we have that the support function transfered to $S^{n}$, with a convenient choice of orientation, tends to $-\infty$ on both ends of $M$. Thus $f$ satisfies $\lim _{q \rightarrow \pm p} f(q)=-\infty$.

REMARK 2.4. If we know the explicit expresion of the generating curve $C$, we can write explictly the function $f$. For instance, in the case of a rotation hypersurface $x: M^{3} \rightarrow$ $\boldsymbol{R}^{4}$ with $H_{2}=0$, we know that the generating curve $C$ is given by

$$
y=1+\frac{x_{1}^{2}}{4} .
$$

A simple computation shows that the support function transfered to $S_{1}^{n}$, i.e., $\langle x, N\rangle \circ$ $g^{-1}=f$ is given by

$$
f(z)=\frac{1-2 z^{2}}{\sqrt{1-z^{2}}}, \quad z=g \circ x_{1}=\frac{x_{1}}{\sqrt{4+\left(x_{1}\right)^{2}}} .
$$

Since $f$ is a radial function, one can easily check, by using the expression of the Laplacian for radial functions (see, for instance, Sakai [13], p. 263) that

$$
\tilde{\Delta} f+3 f=0
$$

as it should be.

Lemma 2.5 below follows an argument of do Carmo and Silveira [4].

LEMMA 2.5. Given finitely many points $p_{1}, \ldots, p_{k} \in S_{1}^{n}$, there exists a domain $W \subset$ $S_{1}^{n}$ that omits neighborhoods $U_{i} \subset S_{1}^{n}$ of $p_{i}, i=1, \ldots, k$, and satisfies $\lambda_{1}(W)=n$. 
Proof. For each $p_{i}$, make a rotation of $S_{1}^{n}$ so that $p_{i}=(0, \ldots, 0,1)$. Let $D_{i}$ and $f_{i}$ be the domain and the function given by Lemma 2.3. Set $h=\sum_{i} f_{i}$ and define $W$ as a connected component of the set $\left\{p \in S_{1}^{n} ; h \geq 0\right\}$.

We recall that a hemisphere $H$ of $S_{1}^{n}$ has eigenvalue $n$ and that, of all domains in $S_{1}^{n}$ with the same area, the spherical cap has the smallest eigenvalue. Since $D_{1} \cap D_{2} \neq \emptyset$, the set $\{p \in$ $\left.S_{1}^{n} ; f_{1}+f_{2} \geq 0\right\}$ is not empty. Thus a connected component $D_{12}$ of $\left\{p \in S_{1}^{n} ; f_{1}+f_{2} \geq 0\right\}$ has eigenvalue $n$ with eigenfunction $f_{1}+f_{2}$. By the above minimization property,

$$
A\left(D_{12}\right)>A\left(H \subset S_{1}^{n}\right),
$$

where $A(\quad)$ denotes the area of the enclosed domain. By the same token, $A\left(D_{i}\right)>A(H)$, $i=1, \ldots, k$. Thus $D_{12} \cap D_{3} \neq \emptyset$, and an induction shows that $A(W)>A(H)$. This shows that $W$ is not empty. Clearly, $\lambda_{1}(W)=n$, and $h$ is the first eigenfunction of $W$. Since $\lim _{p \rightarrow p_{i}} f_{i}=-\infty, W$ omits neighborhoods $U_{i}$ of $p_{i}$, as we desired.

PROOF OF THEOREM 1.1. The proof uses some recent results of [6] on finite total curvature, complete hypersurfaces of $n$-dimensional euclidean spaces. We assume the existence of an immersion $x: M^{n} \rightarrow \boldsymbol{R}^{n+1}$ as in Theorem 1.1. Since $x$ is proper, has finite total curvature, and $H_{n} \neq 0$ everywhere, Theorems 1.1 and 4.1 of [6] imply that there exist a compact manifold $\bar{M}$ and points $q_{1}, \ldots, q_{k} \in \bar{M}$ such that $M$ is diffeomorphic to $\bar{M}-\left\{q_{1}, \ldots, q_{k}\right\}$ and the Gauss map extends to a homeomorphism $\bar{g}: \bar{M} \rightarrow S_{1}^{n}$. Set $p_{i}=\bar{g}\left(q_{i}\right), i=1, \ldots, k$. Let $W \subset S_{1}^{n}$ be the domain, given by Lemma 2.5, that omits neighborhoods $U_{i}$ of $p_{i}$ and is such that $\lambda_{1}(W)=n$. Let $W^{\prime} \supsetneq W$ be a domain in $S_{1}^{n}$ that still omits neighborhoods of $p_{i}$, and set $D=g^{-1}\left(W^{\prime}\right)$. Since $g$ is bijective and $\lambda_{1}(g(D))<n$, we conclude, by Theorem B, that $D$ is unstable. This contradicts the assumption and completes the proof.

EXAMPLE. The following example shows that the hypothesis of stability in Theorem 1.1 cannot be dropped. As mentioned in [1], the hypersurface $M$ in $\boldsymbol{R}^{4}$ generated by the rotation of the parabola $h(z)=1+z^{2} / 4$ around the $z$-axis is a nonstable complete hypersurface with $H_{2}=0$ and $H_{3} \neq 0$ everywhere. By using the orthogonal parametrization $x: M \rightarrow \boldsymbol{R}^{4}$, it is represented as

$$
x(z, \theta, \varphi)=(h \cos \theta \sin \varphi, h \sin \theta \sin \varphi, h \cos \theta, z),
$$

from which we can easily compute that $|A|^{3}=(27 / 8) f^{-9 / 2}$, and that

$$
\int_{M}|A|^{3} d M=\frac{27}{2} \pi^{2}
$$

Thus $M$ has finite total curvature, and this proves our claim.

\section{REFERENCES}

[ 1 ] H. Alencar, M. do CARmo And M. F. Elbert, Stability of hypersurfaces with vanishing $r$-mean curvatures in euclidean spaces, J. Reine Angew. Math. 554 (2003), 201-216.

[ 2 ] J. L. Barbosa And M. P. Do CARmo, On the size of a stable minimal surface in $\boldsymbol{R}^{3}$, Amer. J. Math. 98 (1976), 515-528. 
[ 3 ] J. L. Barbos A And A. G. Colares, Stability of hypersurfaces with constant $r$-mean curvature, Ann. Global Anal. Geom. 15 (1997), 277-297.

[4] M. do Carmo and A. DA Silveira, Globally stable complete minimal surfaces in $\boldsymbol{R}^{3}$, Proc. Amer. Math. Soc. 79 (1980), 345-346.

[ 5 ] M. do Carmo And C. K. Peng, Stable complete minimal surfaces in $\boldsymbol{R}^{3}$ are planes, Bull. Amer. Math. Soc. (N.S.) 1 (1979), 903-906.

[6] M. Do CARmo And M. F. Elbert, Complete hypersurfaces in Euclidean spaces with finite total curvature, Preprint, (2003).

[ 7 ] D. Fischer-Colbrie And R. Schoen, The structure of complete stable minimal surfaces on 3-manifolds of non-negative scalar curvature, Comm. Pure Appl. Math. 33 (1980), 199-211.

[ 8 ] J. Hounie AND M. L. Leite, The maximum principle for hypersurfaces with vanishing curvature functions, J. Differential Geom. 41 (1995), 247-258.

[ 9 ] J. Hounie AND M. L. Leite, Two-ended hypersurfaces with zero scalar curvature, Indiana Univ. Math. J. 48 (1999), 867-882.

[10] J. Hounie AND M. L. LeITE, Uniqueness and non-existence theorems for hypersurfaces with $H_{r}=0$, Ann. Global Anal. Geom. 17 (1999), 397-407.

[11] R. REILLY, Variational properties of functions of the mean curvature in space forms, J. Differential Geom. 8 (1973), 465-477.

[12] H. Rosenberg, Hypersurfaces of constant curvature in space forms, Bull. Sci. Math. 117 (1993), $211-239$.

[13] T. SAKAI, Riemannian Geometry, Transl. Math. Monogr. 149, American Mathematical Society, Providence, RI, 1996.

[14] M. Traizet, On the stable surface of constant Gauss curvature in space forms, Ann. Global Anal. Geom. 13 (1995), 141-148.

Instituto de Matemtica Pura e AplicADA

ESTRADA DONA CASTORINA 110

CEP 22460-320

RIO DE JANEIRO, RJ

BRASIL

E-mail address: manfredo@impa.br
UNIVERSIDADE FEDERAL DO RIO DE JANEIRO

INSTITUTO DE MATEMÁTICA

CX. Postal 68530, CEP 21941-590

RIO DE JANEIRO, RJ

BRASIL

E-mail address: elbert@impa.br 